\title{
Effects of Climate Change on Trait-Based Dynamics of a Top Predator in Freshwater Ecosystems
}

\author{
Yngvild Vindenes, ${ }^{1, \star}$ Eric Edeline, ${ }^{2}$ Jan Ohlberger, ${ }^{1}$ Øystein Langangen, ${ }^{1}$ Ian J. Winfield, ${ }^{3}$ \\ Nils C. Stenseth, ${ }^{1}$ and L. Asbjørn Vøllestad ${ }^{1}$ \\ 1. Centre for Ecological and Evolutionary Synthesis (CEES), Department of Biosciences, University of Oslo, P.O. Box 1066 Blindern, \\ NO-0316 Oslo, Norway; 2. Université Pierre et Marie Curie-Paris 6, Unité Mixte de Recherche 7618 Biogéochimie et Écologie des \\ Milieux Continentaux, 46 rue d’Ulm, 75230 Paris cedex 05, France; 3. Lake Ecosystems Group, Centre for Ecology and Hydrology, \\ Lancaster Environment Centre, Library Avenue, Bailrigg, Lancaster, Lancashire LA1 4AP, United Kingdom
}

Submitted April 15, 2013; Accepted August 23, 2013; Electronically published January 14, 2014

Online enhancements: appendixes, $\mathrm{R}$ code file.

\begin{abstract}
Aвstract: Predicted universal responses of ectotherms to climate warming include increased maximum population growth rate and changes in body size through the temperature-size rule. However, the mechanisms that would underlie these predicted responses are not clear. Many studies have focused on proximate mechanisms of physiological processes affecting individual growth. One can also consider ultimate mechanisms involving adaptive explanations by evaluating temperature effects on different vital rates across the life history and using the information in a population dynamical model. Here, we combine long-term data for a top predator in freshwater ecosystems (pike; Esox lucius) with a stochastic integral projection model to analyze concurrent effects of temperature on vital rates, body size, and population dynamics. As predicted, the net effect of warming on population growth rate (fitness) is positive, but the thermal sensitivity of this rate is highly size- and vital rate-dependent. These results are not sensitive to increasing variability in temperature. Somatic growth follows the temperature-size rule, and our results support an adaptive explanation for this response. The stable length structure of the population shifts with warming toward an increased proportion of medium-sized but a reduced proportion of small and large individuals. This study highlights how demographic approaches can help reveal complex underlying mechanisms for population responses to warming.
\end{abstract}

Keywords: climate change, structured populations, integral projection model, Esox lucius, stochastic dynamics, temperature-size rule.

\section{Introduction}

The global climate is changing rapidly (IPCC 2007), and understanding the mechanisms behind population responses to climate change is currently an important task for biologists (Stenseth et al. 2002; Parmesan 2006; Williams et al. 2008). Climate change is characterized by a

\footnotetext{
* Corresponding author; e-mail: yngvild.vindenes@ibv.uio.no.
}

Am. Nat. 2014. Vol. 183, pp. 243-256. (C) 2014 by The University of Chicago. 0003-0147/2014/18302-54612\$15.00. All rights reserved.

DOI: $10.1086 / 674610$ gradual increase in the mean temperature. It is bringing about other changes such as milder, wetter, and stormier winters in northern Europe and an increased frequency of extreme events (IPCC 2007). Predicted responses to warming across taxa include shifts in species ranges toward higher latitudes and altitudes (Walther et al. 2002), shifts in phenology (Visser and Both 2005), and a reduced body size (Daufresne et al. 2009; Gardner et al. 2011).

Observed responses to climate change reflect both individual- and population-level processes that are also affected by population structure. Vital rates like survival, fecundity, and somatic growth rate can respond differently to the same climate variable (e.g., van de Pol et al. 2010). Moreover, the same vital rate can show different responses to climatic changes depending on age, sex, body size, or other individual state variables (Coulson et al. 2001) However, the majority of current studies of climate change responses either link population responses directly to climatic variables (e.g., Boylan and Adams 2006; Friedland and Todd 2012) or consider only one or a few vital rates like survival or fecundity (e.g., Crozier et al. 2008; Jonsson and Jonsson 2009). The former approach cannot provide insights of the underlying mechanisms, while the latter approach cannot predict population level-responses. Demographic approaches are useful because they link the individual-level and population-level processes (Caswell et al. 1997). Here we use a stochastic integral projection model and long-term data from a length-structured population of pike Esox lucius, a top predator in freshwater ecosystems, to consider concurrent effects of warming on vital rates, population growth (mean and variability), and the stable length distribution. As a top predator, pike responses to climate change may induce cascading effects with consequences for other species (Estes et al. 2011).

Most demographic models to date that study consequences of climate change have been applied to popula- 
tions with discrete structure of age or stage, using matrix models (e.g., Jenouvrier et al. 2009; Hunter et al. 2010; Jonzén et al. 2010; van de Pol et al. 2010, 2011). Continuous structure due to body size has been studied using detailed physiologically structured population models (Metz and Diekmann 1986), but the method has been applied to only very few wild populations (e.g., Ohlberger et al. 2011a). Physiologically structured population models have a continuous timescale and a high level of complexity, and the data requirements are therefore difficult to meet for many wild study populations. Obtaining continuoustime individual observations is often not possible, and much effort may be required to obtain even annual data (Clutton-Brock and Sheldon 2010). Integral projection models (IPMs; Easterling et al. 2000) are based on continuous state structure (e.g., due to body size) but discrete time steps and may therefore provide a useful alternative for analysis of size-structured populations in the wild. In the simplest form of an IPM, individuals are classified according to one continuous state variable, and the vital rates are modeled as a function of the state variable using regression techniques (Ellner and Rees 2006). As a continuous-state analogue to matrix models (Caswell 2001), IPMs retain all the analytical advantages of this model class (Ellner and Rees 2006).

Body size is a fundamental biological trait known to affect several important life-history traits (Calder 1984; Bonner 2006). Survival probability generally increases with size, largely because individuals become less vulnerable to predation and better able to cope with stressful conditions (Roff 2002). Fecundity also often increases with size, in particular in ectotherms and plants, where size can be a direct determinant of the number of eggs or seeds an individual can produce (Roff 2002). Thus, there are often clear benefits of being large in terms of increased survival and fecundity, but getting large requires energy that induces costs associated with growth. Trade-offs between the vital rates of growth, fecundity, and survival determine the organism's schedule of reproduction over its lifetime and are at the heart of the theory of life-history evolution (Charlesworth 1994; Stearns 2000; Roff 2002). Extrinsic factors such as temperature can affect one or more of the vital rates and thereby induce changes in the other rates, potentially giving rise to evolutionary changes in life history (Clarke 2003).

The temperature-size rule (TSR) of ectotherms describes a commonly observed phenotypic response of organisms to the thermal environment during ontogeny, where warming increases rates of growth and development, but individuals reach a reduced final body size (Atkinson 1994, 1996). In addition to this change in growth pattern, average mortality and population growth rates are also predicted to increase with temperature in ectotherms (Savage et al. 2004; Peck et al. 2009). Proposed mechanisms of the TSR often focus on the proximate causes such as biochemical reactions fueling maintenance and reproduction processes (Brown 2004). The TSR may also reflect adaptive life-history responses to extrinsic temperature effects on survival and/or fecundity as a function of body size (Kozlowski et al. 2004; Arendt 2010), but few studies consider simultaneous thermal responses of size-specific survival and fecundity. A demographic approach can elucidate such patterns, by revealing concurrent effects of climate change on vital rates, key biological traits (like body size), and population dynamical parameters.

Here, we develop and analyze a temperature-driven stochastic IPM for a population of pike in the lake Windermere in the United Kingdom, using a unique longterm data set of individual demographic rates and morphological traits. In this study, we aim to (i) estimate the effects of temperature on each vital rate as a function of body length, through regression modeling of long-term data, and evaluate whether they are consistent with common observed patterns in ectotherms; (ii) investigate how properties of the stochastic population growth are affected by changes in the mean and variability of the temperature process; (iii) disentangle the thermal sensitivity of the population growth rate (fitness) by decomposing it into contributions across different vital rates and body length; (iv) evaluate effects of warming on the length structure in the population; and (v) discuss whether the vital rate and population responses are consistent with predictions from general life-history theory and whether the results support an adaptive explanation for the TSR.

\section{Methods \\ Study System and Data}

This study utilizes data collected at Windermere, which is situated in the English Lake District, United Kingdom $\left(54^{\circ} 22^{\prime} \mathrm{N}, 2^{\circ} 56^{\prime} \mathrm{W}\right.$; altitude $\left.39 \mathrm{~m}\right)$. The lake consists of a mesotrophic north basin and a eutrophic south basin with similar fish species composition (Winfield et al. 2008). Data have been collected since the 1940s, covering pike and other major fish populations as well as the abiotic and biotic environment (a review of the history of the study is given by Le Cren 2001). In the northwest United Kingdom, annual mean air temperature is predicted to increase over the next century, under different emission scenarios (Jenkins et al. 2009). Pike prefer shallow water where we can expect similar temperature changes to apply, and the observed surface temperature of Windermere has already shown a marked increase over the recent decades (Winfield et al. 2008).

Scientific monitoring of the pike population was initi- 
Table 1: Overview of the main vital rates as a function of length $x$ and temperature $z$, and their components

\begin{tabular}{ll}
\hline Vital rate & Signification \\
\hline$S(x, z)$ & $\begin{array}{l}\text { Survival probability } \\
B(x, z)=0.5 S_{\mathrm{egg}} M(x, z)\end{array}$ \\
$\begin{array}{l}\text { Fecundity (number of 1-year-olds produced by female), where } M(x, z) \text { is } \\
\text { the egg number and } S_{\text {egg }} \text { is the survival probability from egg to age } 1\end{array}$ \\
$\begin{array}{l}\text { Somatic growth distribution from size } x \text { to size } y \text { next year, mean size } \\
\text { next year is given by } \mu_{\mathrm{g}}(x, z) \text { and variance is } \sigma_{\mathrm{g}}^{2}(x) \text {; individuals are not } \\
\text { allowed to shrink in length } \\
\text { Length distribution of offspring (age 1), lognormal distribution with } \\
\text { mean on absolute scale } \mu_{g 1}(z) \text { and variance on absolute scale } \sigma_{g 1}^{2}\end{array}$ \\
\hline
\end{tabular}

ated in 1944 with a removal program to enhance perch (Perca fluviatilis) numbers and continues today with few changes in methods other than a much reduced sampling effort (Le Cren 2001). Each fall/winter (in most years from mid-October to December), pike have been captured with 64-mm-mesh gill nets in shallow areas of the lake. Captured pike are killed, measured for body length (measured as $\mathrm{cm}$ fork length), weighed $(\mathrm{kg})$, and sexed, and opercular bones are removed for age and length back-calculation, following a method validated for Windermere pike by Frost and Kipling (1959). In this study, we use body length estimates that are measured and back-calculated using catch data for females from 1944 to 1995 (7,939 individuals, 34,440 length estimates in total). Since 1963, data on female reproductive investment (gonad weight, egg number, and egg weight) have also been collected (Frost and Kipling 1967). During the first few years there was an increase in average egg production after a peak in fishing pressure in 1963, followed by a slow decrease (Edeline et al. 2007). Here we use egg number data from the period 1968-2002 (3,634 individuals), corresponding to the period with slow decrease.

Survival probability estimates are based on data from a separate capture-mark-recapture (CMR) study, which is not ongoing today. Before and during the spring spawning period (March-April), individuals were caught in a spring sampling program using 38- and 64-mm-mesh-size gill nets and perch traps, and then weighed, sexed, lengthmeasured, and tagged before release (Kipling and Le Cren 1984; Haugen et al. 2007). The tags were made of monel metal with punched individual numbers, and since 1953 they have been attached to the maxilla bone (upper jaw; Kipling and Le Cren 1984). Some marked individuals were later caught in the spring sampling program, but most were caught (and killed) during the winter fishery (Carlson et al. 2007). Due to reduced sampling effort, the number of pike tagged and recaptured after 1990 has been very low (Haugen et al. 2007). Therefore, we use CMR data from individuals captured in the period 1953-1990 in this study. Data from males, females, and individuals of un- known sex (3,992 individuals in total) are used in order to increase the statistical strength of the estimation procedure.

The four data sets associated with this study are available online (see URL for each data set in the references): (i) fecundity data (Winfield et al. 2013a), (ii) growth data (Winfield et al. 2013b),( iii) survival data (Winfield et al. 2013c), and (iv) lake temperature data (Winfield and Fletcher 2013). Further inquiries may also be made directly to I. J. Winfield.

\section{Model Description}

In this section we describe the integral projection model and its main parameters, while methods for estimating vital rates from data are described in the next section. The model is female based and density independent, with a prereproductive census. We use capital letters to denote the vital rates and projection kernel in the stochastic environment, while lowercase letters are used for the vital rates and kernel in the mean environment. The environment is assumed to follow a stationary process, where climate change may alter both the mean and variability. An overview of the four main vital rate functions entering the model is given in table 1 .

We considered age as a potential state variable in addition to length, but the modeling of fecundity and somatic growth rate (where age data is available) revealed that body length accounts for most of the variation between individuals. The population dynamics (growth rate, stable length structure, reproductive values) were similar in a model including only body length and a model including both age and length as state variables (data not shown). For the somatic growth rate, age did explain some of the variation in addition to length (as younger individuals tend to grow faster at a given length). However, we could capture this variation using a variance function that declines with length.

Annual Mean Surface Temperature. The annual mean sur- 
face temperature, denoted $Z$, is a key climatic variable in this system, and fluctuations in $Z$ between years give rise to some of the environmental stochasticity in the population dynamics. The observed temperature time series is shown in figure Al in appendix A (apps. A-D available online), with an increasing trend over the period 19862001. There was no evidence of temporal autocorrelation (after detrending the period 1986-2003), and we assume $Z \sim N\left(\mu_{z}, \sigma_{z}\right)$ (within any given year, $Z=z$, and we use $z$ to denote temperature). The baseline model is based on the observed mean and standard deviation of the period $1944-1985, \mu_{z}=10.34^{\circ} \mathrm{C}$, and $\sigma_{z}=0.54^{\circ} \mathrm{C}$. In the analysis, we consider effects of changes in both parameters on the vital rates, length structure, and population dynamical parameters.

Life Cycle, Vital Rates, and Projection Function. For each year, we assume that an individual first reproduces, then (if it survives) grows to reach a new body length next year. The survival probability, somatic growth rate, and fecundity all depend on the current length $x$ (in $\mathrm{cm}$ ) of the individual. If the offspring survive from egg to age 1 , they enter the population next year as 1-year-olds, with a length according to a distribution which is independent of parental length. Thus, there are four main vital rate functions that depend on body length $x$ and temperature $z$ : (i) survival probability $S(x, z)$, (ii) fecundity $B(x, z)$ (number of female offspring produced by an individual with length $x$ ), (iii) the length distribution of offspring $F(y ; z)$, and (iv) the length distribution of next year's size given this year's size $G(y ; x, z)$. These four vital rate functions show temporal covariation, some of which is caused by the common temperature. Residual covariation will be modeled from the yearly residuals (see section on vital rate estimation).

The fecundity function can be written as $B(x, z)=$ $0.5 M(x, z) S_{\text {egg }}$, where $M(x, z)$ is the number of eggs and $S_{\text {egg }}$ is the survival probability from egg to 1 year old. The sex ratio of eggs is assumed to be 0.5 . The size of maturity is set to $x_{\min }=38 \mathrm{~cm}$ (the smallest observed reproducing individual in the data set was $40.8 \mathrm{~cm}$ ), so that for $x<$ $x_{\min }$ we have $M(x, z)=0$. The egg survival probability is assumed to follow a model on the form $\operatorname{logit} S_{\mathrm{egg}} \sim N\left(\beta_{\mathrm{egg}}\right.$, $\sigma_{\text {egg }}^{2}$. We do not have the data to estimate the parameters of this function, thus $\beta_{\mathrm{egg}}$ and $\sigma_{\mathrm{egg}}^{2}$ are free parameters in our model. Their values $\left(\beta_{\text {egg }}=0.00019\right.$ and $\sigma_{\text {egg }}^{2}=$ 0.01 ) are chosen so that the mean and variance of the yearly population growth rate from our model (using baseline values of parameters) would be close to the mean and variance from a population density time series (Langangen et al. 2011). The mean egg survival of 0.00019 is comparable to estimates of early survival reported from other pike studies (Wright 1990) and to an earlier estimate of
Windermere pike (Kipling and Frost 1970). We assume that egg survival is independent of temperature. However, since an earlier study indicated a positive effect of temperature on early survival (Craig and Kipling 1983), we also constructed an alternative model including a weak and a strong positive temperature effect on egg survival. The results are shown in appendix $\mathrm{B}$, indicating that the qualitative conclusions of this study are not affected by this assumption.

The offspring ( 1 year old) length distribution $F(y ; z)$ is assumed to be lognormal (as this distribution fitted the observed data well), where the mean $\mu_{g 1}(z)$ depends on temperature and the variance $\sigma_{g 1}^{2}$ is constant. The somatic growth function $G(y ; x, z)$ gives the distribution of next year's length $y$ for a given current length $x$ (the probability of getting a size within the interval $\left[y_{1}, y_{2}\right]$ is given by $\int_{y_{1}}^{y_{2}} G(y ; z, z) \mathrm{d} y$ ). Individuals are not allowed to shrink (in length), so we have $G(y ; x, z)=0$ for $y<x$. For $y \geq x$, $G(y ; x, z)$ follows a truncated lognormal distribution, scaled so that $\int_{0}^{\infty} G(y ; x, z) \mathrm{d} y=1$. The mean of next year's size is given by $\mu_{g}(x, z)$, declining with length and restricted to be nonnegative. The variance of next year's size is given by a function $\sigma_{g}^{2}(x)=\tau_{g} e^{\nu_{g} x}$ that is independent of temperature and declines exponentially with length. Thus, individuals grow to reach an asymptotic length.

The population growth from one year to the next is described by a stochastic projection function (kernel) that depends on the four vital rate functions (Easterling et al. 2000). Here, this function is given by

$$
K(y, x, z)=S(x, z) G(y ; x, z)+B(x, z) F(y ; z),
$$

where $S(x, z) G(y ; x, z)$ is the kernel describing survival and growth of individuals from size $x$ to size $y$, while $B(x$, $z) F(y ; z)$ is the kernel describing production of new individuals of size $y$ by individuals of size $x$. This projection function takes new values each year depending on the random year effects and temperature. Letting $n_{t}(x)$ represent the number (density) of individuals of length $x$ in year $t$, the total population density is $N_{t}=\int_{0}^{\infty} n_{t}(x) \mathrm{d} x$ and the growth of the population to next year is given by $N_{t+1}=\int_{0}^{\infty} n_{t+1}(y) \mathrm{d} y=\int_{0}^{\infty} \int_{0}^{\infty} K_{t}(y, x, z) n_{t}(x) \mathrm{d} x \mathrm{~d} y$.

Calculations for the Mean Environment. The projection function in the mean environment (with temperature equal to the mean $\mu_{z}$ and no stochasticity) is denoted $k(x$, $\left.y, \mu_{z}\right)$. Based on this function we find the expected population growth rate $\lambda$, the stable age/length structure $u(x)$, and the reproductive value $v(x)$ (Haccou et al. 2005; Ellner and Rees 2006). Existence of unique solutions to these functions requires that the model is properly defined and bounded, as described by Ellner and Rees (2006). The stable length structure is scaled so that $\int_{0}^{\infty} u(x) \mathrm{d} x=1$, and the reproductive value function is scaled so that 
$\int_{0}^{\infty} v(x) u(x) \mathrm{d} x=1$. Numerically, we find these parameters by discretizing the projection function (after estimation using regression), in order to obtain a large projection matrix (we use a $300 \times 300$ projection matrix in the calculations for the mean environment) and then use standard methods for eigenvalue analysis of matrix models (Caswell 2001).

The sensitivity of $\lambda$ to a small perturbation of the projection kernel near a point $[x, y]$ is given by $v(y) u(x)$ (Ellner and Rees 2006), using our scaling of these functions. Here we are interested in the sensitivity of $\lambda$ (fitness) to the mean temperature $\mu_{z}$. This sensitivity can be decomposed into contributions from each vital rate and length; that is,

$$
\begin{aligned}
\frac{\mathrm{d} \lambda}{\mathrm{d} \mu_{z}}= & \iint \frac{\partial \lambda}{\partial k\left(y, x, \mu_{z}\right)} \frac{\partial k\left(y, x, \mu_{z}\right)}{\partial \mu_{z}} \mathrm{~d} y \mathrm{dx} \\
= & \int v(y) \int u(x)\left[\frac{\partial s\left(x, \mu_{z}\right)}{\partial \mu_{z}} g\left(y, x, \mu_{z}\right)\right. \\
& +\frac{\partial g\left(y, x, \mu_{z}\right)}{\partial \mu_{z}} s\left(x, \mu_{z}\right)+\frac{\partial b\left(x, \mu_{z}\right)}{\partial \mu_{z}} f\left(y, \mu_{z}\right) \\
& \left.+\frac{\partial f\left(y, \mu_{z}\right)}{\partial \mu_{z}} b\left(x, \mu_{z}\right)\right] \mathrm{d} x \mathrm{~d} y .
\end{aligned}
$$

The innermost integral contains contributions for given length $x$ through each of the four vital rate functions. The first term is the contribution through survival, the second through somatic growth, the third through fecundity, and the fourth through offspring growth. Together, these four functions describe how temperature affects the population growth rate through the different parts of the life history and length ranges of pike.

Using the same approach we also consider the sensitivity of fitness to changes in length $x$ (at a given temperature $\mu_{z}$ ), which is given by

$$
\begin{aligned}
\frac{\mathrm{d} \lambda}{\mathrm{d} x}= & \int v(y) u(x)\left[\frac{\partial s\left(x, \mu_{z}\right)}{\partial x} g\left(y, x, \mu_{z}\right)\right. \\
& \left.+\frac{\partial g\left(y, x, \mu_{z}\right)}{\partial x} s\left(x, \mu_{z}\right)+\frac{\partial b\left(x, \mu_{z}\right)}{\partial x} f\left(y, \mu_{z}\right)\right] \mathrm{d} y .
\end{aligned}
$$

Because we assume that the offspring length distribution is independent of parental length, there is no contribution from the sensitivity of this vital rate function in the above expression.

We have provided the $\mathrm{R}$ code, available online, for the decomposition of the thermal sensitivity of $\lambda$ (eq. [2]), using the vital rate functions of this study. These functions can easily be modified to other organisms and life histories. In the same R script we also show how to construct the temperature-specific projection function from the vital rates, and how to calculate $\lambda, u(x)$, and $v(x)$ from the projection function.

Stochastic Population Growth and Environmental Variance. While most of our results apply to mean environment, we also analyzed a stochastic model to test whether (i) the decomposition of the thermal sensitivity of $\lambda$ (eq. [2]) is a good approximation also for the stochastic growth rate $\lambda_{s}$, (ii) the stochastic dynamics are well described by a diffusion approximation and thus that the stochastic growth rate and environmental variance are sufficient to capture the long-term dynamics of the system, and (iii) changes in the variability of the temperature process have small effects compared to changes in the mean.

To study properties of the stochastic population growth in response to temperature changes, we consider the dynamics of the total reproductive value $V_{t}=$ $\int_{0}^{\infty} n_{t}(x) v(x) \mathrm{d} x$, where $v(x)$ is calculated for the mean environment as described above. In contrast to the total population size $N_{t}$, which shows transient dynamics due to fluctuations in the length structure, the dynamics of $V_{t}$ are approximately Markovian and can be described by a diffusion approximation with only two parameters: the stochastic growth rate $r_{s}$ and the environmental variance $\sigma_{e}^{2}$ (Engen et al. 2007; Vindenes et al. 2011). The environmental variance describes the variance of the population growth on $\log$ scale, $\sigma_{e}^{2} \approx \operatorname{Var}(\Delta \ln V)$ (Engen et al. 2007). The diffusion approximation of $\ln V_{t}$ is defined by infinitesimal mean $r_{s}$ and infinitesimal variance $\sigma_{e}^{2}$ (Lande and Orzack 1988).

To calculate $r_{s}$ and $\sigma_{e}^{2}$ in this model, we first generate a large number $(T=5,000)$ of projection matrices based on the projection function (using $50 \times 50$ matrices), where each realization corresponds to a new time step. These are then used to project the population density over time, starting from an arbitrary initial length distribution (we used $n_{0}(x)=100 u(x)$, that is, a density of 100 individuals distributed according to the stable length distribution in the mean environment). The reproductive value function $v(x)$ calculated for the mean projection function is used to generate a time series of the total reproductive value $V_{t}(t=1, \ldots, T)$. Finally, we estimate the stochastic growth rate as $\hat{r}_{s}=T^{-1}\left(\ln V_{T}-\ln V_{1}\right)$, and the environmental variance as $\hat{\sigma}_{e}^{2}=(T-1)^{-1} \sum_{j=2}^{T} \times$ $\left(\ln V_{j}-\ln V_{j-1}-\hat{r}_{s}\right)^{2}$ (Engen et al. 2007).

By the small-noise approximation of Tuljapurkar

1 Code that appears in the American Naturalist is provided as a convenience to the readers. It has not necessarily been tested as part of the peer review. 
(1990), the stochastic growth rate is $r_{s} \approx \ln \lambda-1 / 2 \lambda^{-2} \sigma_{e}^{2}$, where $\lambda$ is calculated for the mean projection function across environments (Rees and Ellner 2009). In general, this is not the same as $\lambda$ calculated for the mean environment, due to Jensen's inequality. Using the small-noise approximation, the sensitivity of the stochastic growth rate to the mean temperature is given by

$$
\frac{\mathrm{d} r_{s}}{\mathrm{~d} \mu_{z}} \approx\left(\frac{1}{\lambda}+\frac{\sigma_{e}^{2}}{\lambda^{3}}\right) \frac{\mathrm{d} \lambda}{\mathrm{d} \mu_{z}}-\frac{1}{2 \lambda^{2}} \frac{\mathrm{d} \sigma_{e}^{2}}{\mathrm{~d} \mu_{z}} .
$$

In appendix $\mathrm{D}$, we show that in this particular model the latter two terms are very small compared to the first and that the decomposition of the temperature sensitivity in equation (2) therefore provides a good approximation also for the sensitivity of stochastic growth rate to mean temperature.

\section{Estimation of Vital Rate Functions}

Four of the functions entering the model $\left(M(x, z), \mu_{g}(x\right.$, $z), \sigma_{g}^{2}(x)$, and $\left.\mu_{g 1}(z)\right)$ were estimated from the three data sets using generalized linear mixed models (Zuur et al. 2009). The constant variance in offspring length $\sigma_{g 1}^{2}$ was also estimated from the length data. All analyses were done with the program R (R Development Core Team 2011), using the packages nlme (Pinheiro et al. 2011) and lme4 (for survival probability; Bates et al. 2011). All models include a random year effect, in order to estimate the residual environmental variance and covariance among the vital rates. We also included a fixed year effect to account for temporal trends caused by other factors than temperature (see Edeline et al. 2007) that are not the focus of this study. In the subsequent analysis, we used the average year for each vital rate in the models.

For each vital rate we fitted a set of candidate models with different combinations of fixed effects of temperature, year, and length, including second-order effects and interaction effects. The most parsimonious model was selected based on the lowest value of Akaike's Information Criterion (AIC), and the AIC difference to the next model was always $>2$ (Burnham and Anderson 2002). The candidate models were fitted with maximum likelihood estimation, and the final model was refitted using restricted maximum likelihood. In the somatic growth model, an exponential variance function was included in order to estimate the parameters of $\sigma_{g}^{2}(x)$. In the model for egg number $M(x, z)$, an exponential variance function was also included, to account for heteroscedasticity. We also checked for potential problems of multicolinearity between the fixed temperature and year effect, as temperature and year had a relatively high correlation of 0.67 for the fecundity data (for the two other data sets, spanning dif- ferent years, the correlation was $<0.2$ ). The estimated temperature effect in the model for egg number did not change much if year was excluded, and the variance inflation factor (defined as $1 /\left(1-R_{j}^{2}\right)$, where $R_{j}^{2}$ is the multiple $R^{2}$ of an ordinary least squares regression of effect $j$ on the other effects in the model) was low both for the year effect (1.48) and for the temperature effect (1.71), indicating that multicolinearity is not very high.

The survival probability model required a more complex estimation procedure, in which we extended the method of Carlson et al. (2007) to avoid a potential source of bias. This method is described in detail in appendix C. The goal was to estimate the survival probability between the first capture in spring and the next capture event, in autumn (assuming a winter survival of 1). Therefore, following Carlson et al. (2007) we first assigned a survival probability of 1 (for surviving the first summer) to all individuals that are ever recaptured again. The individuals that are never seen again were assigned a survival probability of 0 . While these have certainly died at some point (Windermere is a closed system, so practically all individuals that are alive will eventually be recaptured), the time of death could be a later year than the capture year, and if so, the body length would likely be larger and the temperature would be different. To correct for this potential bias due to uncertainty in time of death, we used the somatic growth model of this study together with the annual recapture probabilities given by Carlson et al. (2007) in a Monte Carlo iteration procedure (app. C). The data were resampled to assign new times of death to the individuals that were never seen again after the first capture. At each iteration, the survival model was refitted, and then the new model was used in the next iteration. The survival function rapidly converged toward a stationary distribution, and the final model was based on the mean effects from 1,000 iterations after a burn-in period of 100 (see details in app. C).

For the final survival model we imposed a constant survival (constant as function of length, not temperature) for large individuals, although the fitted model suggested a decline with length. This decline may occur if larger individuals have a lower capture probability, which is often the case with gill nets (Lagler 1978). We do not know the length-specific gill net sensitivity for this pike population but believe that such a reduction in catch probability of large individuals likely explains much of the decline in apparent survival (for smaller individuals both $34-\mathrm{mm}$ and 64-mm gill nets are used, so that estimated survival at small and medium size is probably not as affected by gill net sensitivity as at large size). It could also be that larger individuals experience an increased mortality, for instance due to senescence (although this may be unlikely in fish, where fecundity is usually increasing with size). To test the consequences of assuming a constant survival of large in- 

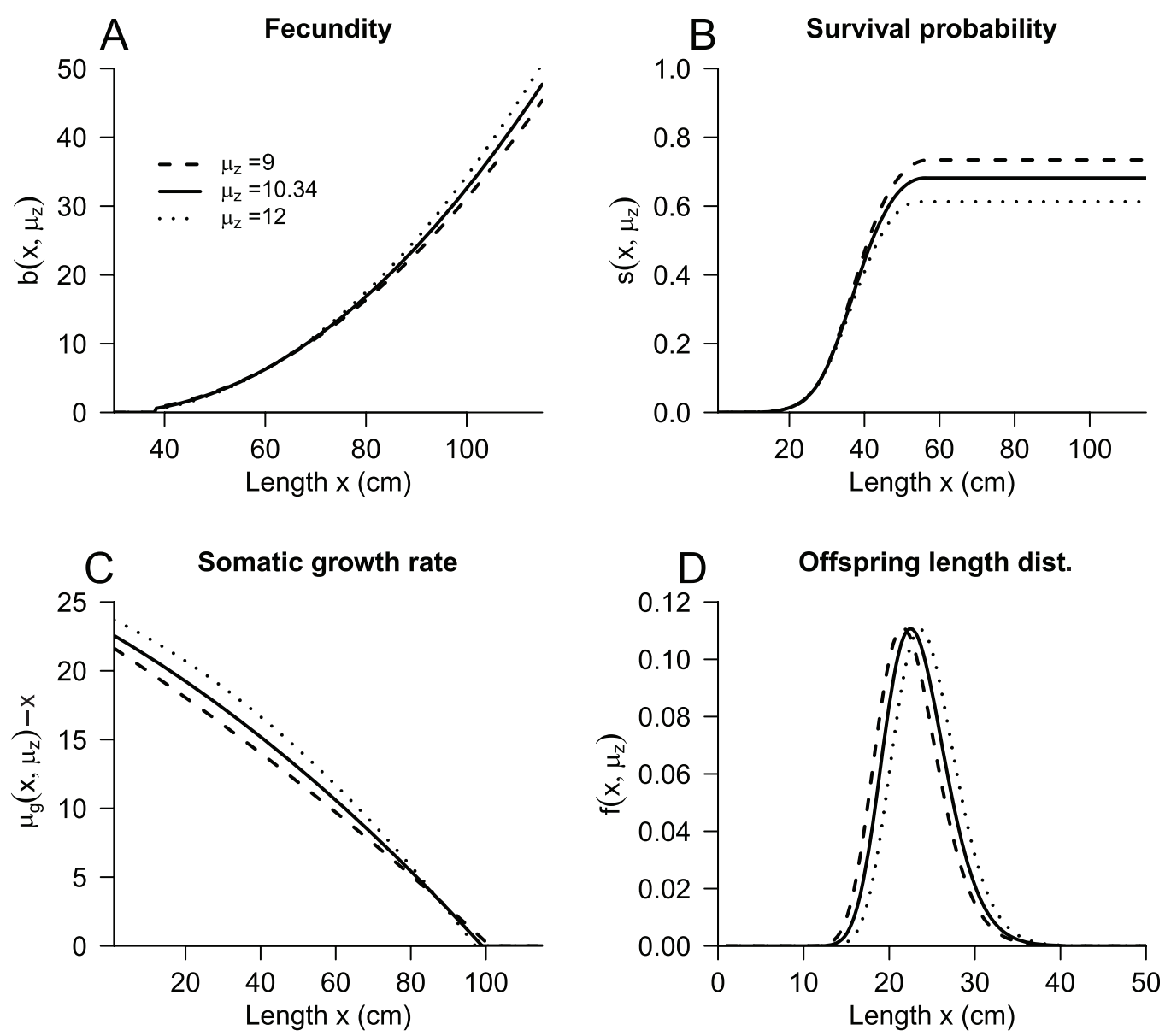

Figure 1: Vital rate functions in the mean environment, for three values of the mean temperature $\mu_{z}$. A, Fecundity (mean number of 1year-old female offspring produced by a female), the minimum length of reproduction is $38 \mathrm{~cm}$. $B$, Survival probability from one year to the next as a function of length. $C$, Somatic growth rate $(\mathrm{cm} /$ year) as a function of length. Next year's length $y$ of an individual of length $x$ follows a truncated lognormal distribution with mean $\mu_{g}\left(x, \mu_{z}\right)(y \geq x)$. D, Density distribution (lognormal) for the length of a 1-yearold offspring, where the mean depends on mean temperature.

dividuals, we ran the analysis using the fitted survival curve that declines for large lengths, and results were qualitatively similar, except for the shape of the reproductive value function (not shown). Thus, our model results are robust to uncertainty in survival of large individuals, and this is likely because only a small proportion of individuals reach large sizes.

After all of the vital rate functions had been estimated, we used the random year effects to estimate a variance/ covariance matrix $\Sigma y$. The covariances in $\Sigma y$ were estimated using all pairwise complete pairs of year effects for the vital rates data, while the variances of the year effects (diagonal of $\Sigma y$ ) were estimated using the entire length of the time series for each vital rate. This matrix does not describe the actual covariance between vital rates, which also depends on the common temperature effect but accounts for the residual covariance. For the stochastic sim- ulations, the random-year effects were drawn assuming a multivariate Gaussian distribution with mean 0 and variance/covariance given by $\Sigma y$.

\section{Results}

\section{Effects of Temperature on Vital Rates}

All vital rates are highly dependent on length, and respond differently to temperature (fig. 1). For small individuals, temperature has a negative effect on fecundity but a positive effect on survival (fig. $1 \mathrm{~A}, 1 \mathrm{~B}$ ). The picture is opposite for large individuals, where temperature has a positive effect on fecundity but a negative effect on survival (fig. $1 A, 1 B)$. The somatic growth is positively affected by temperature except for the largest individuals. The shape of the curve as a function of length is also changing slightly 
with temperature, to be more linear at colder temperatures (fig. 1C). The mean of the offspring length distribution also depends positively on temperature (fig. $1 D$ ), so that after years with a high temperature, offspring enter next year's population at a larger size. The fixed effects of the final regression models entering the vital rate functions are given in table 2 , while table 3 shows the variancecovariance matrix for the random year effects.

The somatic growth of pike follows the temperaturesize rule, as with increasing temperature individuals grow faster at young age but reach a smaller asymptotic length than in colder temperatures (fig. 2). The overall mean length in the population $\left(\int x u(x) \mathrm{d} x\right)$ increases slightly from $26.3 \mathrm{~cm}$ at $\mu_{z}=9$ to $28.4 \mathrm{~cm}$ at $\mu_{z}=12$.

\section{Effects of Temperature on Population Growth and Structure}

From the projection function in the mean environment, $k\left(y, x, \mu_{z}\right)$, we calculated the population growth rate, stable structure, and reproductive values for different temperatures (fig. 3). The population growth rate increases nearly linearly with $\mu_{z}$ (fig. $3 \mathrm{~A}$ ), showing that the positive effects of temperature on vital rates overwhelm the negative effects. The reproductive value increases with length at all temperatures (fig. 3B), and at colder temperatures, the increase happens slightly faster for small individuals.

In general, the stable length distribution reflects a higher proportion of small than large individuals, as expected (fig. $3 C, 3 D)$. The function declines with length in a nonlinear fashion because we have a model with discrete time, and each local peak corresponds to an age class. For large lengths, this age pattern is less visible as ages overlap more. Warming shifts the stable length distribution toward a lower proportion of both small and large individuals (the decline is largest for large individuals) and an increased proportion of medium-sized individuals $(40-90 \mathrm{~cm})$. This happens because of the increased somatic growth rate of small individuals in combination with the reduced survival of larger individuals (fig. 1). Within each age class (the local peaks in the stable length distribution) the mean
Table 3: Estimated variance/covariance matrix $\Sigma_{y}$ for the random-year effects of the vital rate functions estimated using glmm (see table 2)

\begin{tabular}{lrrrr}
\hline & $\mu_{g}(x, z)$ & $\sqrt{M(x, z)}$ & $\operatorname{logit} S(x, z)$ & $\mu_{g 1}(z)$ \\
\hline$\mu_{g}(x, z)$ & .397 & 3.947 & -.002 & .465 \\
$\sqrt{M(x, z)}$ & 3.947 & 77.053 & .061 & 3.223 \\
$\operatorname{logit} S(x, z)$ & -.002 & .061 & .009 & .015 \\
$\mu_{g 1}(z)$ & .465 & 3.223 & .015 & .910 \\
\hline
\end{tabular}

length increases but only for small to intermediate lengths where the somatic growth depends positively on temperature (fig. 1C).

\section{Decomposition of the Thermal and Length Sensitivity of Fitness}

Calculated directly (by small perturbation of $\mu_{z}$ in $k[y, x$, $\left.\left.\mu_{z}\right]\right)$, the temperature sensitivity of $\lambda$ at $\mu_{z}=10.34$ is $\mathrm{d} \lambda /$ $\mathrm{d} \mu_{z} \approx 0.01014$. According to equation (2), we decomposed this sensitivity into contributions from each vital rate as a function of length. These are shown in figure 4A. Summing up these contributions also gives a total sensitivity of 0.01014 , close to the direct estimate. Because $\lambda$ is an approximately linear function of $\mu_{z}$ (fig. $3 A$ ), the sensitivity decomposition gives similar results for a large range of mean temperatures (we checked this for $\mu_{z}=12$ and $\mu_{z}=8$ ).

The decomposition shows that the thermal sensitivity of $\lambda$ depends on both vital rate and length (fig. $4 A$ ). For small pike (length $<\sim 37 \mathrm{~cm}$, largely corresponding to immature individuals) the total sensitivity contributions are positive, due to the positive temperature effect on survival and somatic growth (fig. 1). For medium-sized individuals (length $>\sim 37 \mathrm{~cm}$ ), the thermal sensitivity becomes negative, mostly because of the negative temperature effect on survival but also from a negative effect of temperature on fecundity. The temperature effect then turns positive again for large individuals (lengths $>$ $\sim 75 \mathrm{~cm}$ ), due to positive effects on fecundity, somatic growth, and offspring growth (fig. 1). As the stable dis-

Table 2: Estimated fixed effects of the four generalized linear mixed models used to estimate the vital rate functions (see variance/ covariance matrix for the random-year effects in table 3)

\begin{tabular}{lccrrrrr}
\hline & Intercept & Length & Length & Temp & Length : temp & Year & Length ${ }^{2}:$ temp $^{2}$ \\
\hline$\sqrt{M(x, z)}$ & $1,184.138(395.239)$ & $5.659(1.096)$ & & $-15.064(7.239)$ & $.247(.102)$ & $-.637(.200)$ & \\
$\operatorname{logit} S(x, z)$ & $74.892(3.569)$ & $.510(.038)$ & $-.004(.000)$ & $.193(.175)$ & $-.007(.003)$ & $-.045(.001)$ & \\
$\mu_{g}(x, z)$ & $-64.144(12.831)$ & $.685(.167)$ & $.002(.000)$ & $.685(.185)$ & $-.016(.003)$ & $.041(.007)$ & $-.0003(.000)$ \\
$\mu_{g 1}(z)$ & $-63.373(21.98)$ & & & $.662(.270)$ & & $.041(.012)$ & \\
\hline
\end{tabular}

Note: Each effect is given with standard error in parentheses; for the survival model the standard errors are calculated from 1,000 iterations (see details in app. C). The constant SD of the offspring distribution is $\sigma_{g 1}=3.72$, and the parameters of the variance function for somatic growth are $\tau_{g}=13.82$ and $\nu_{g}=-0.0077$; temp $=$ temperature. 


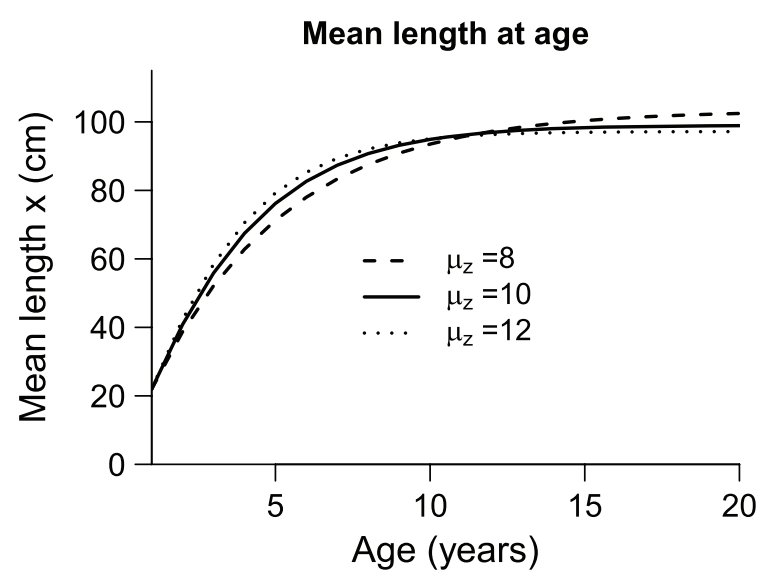

Figure 2: Mean length at age according to the somatic growth model, at three different mean temperatures (starting from the mean length at age 1 for each temperature).

tribution declines to zero, the thermal sensitivity also approaches zero with large length.

Thermal sensitivity of $\lambda$ is negative for medium lengths. However, the sensitivity of $\lambda$ to length was always nonnegative, and largest for the smallest pike (fig. $4 B$ ). Because survival as well as fecundity are increasing functions of length for all temperatures (survival is constant for large individuals), it is always beneficial to increase in size. This is reflected in the reproductive value, which is always increasing (fig. $3 B$ ). The sensitivity of $\lambda$ to length is higher for small individuals, because they constitute the highest proportion of the population (fig. $3 C, 3 D$ ). As the temperature increases, $\lambda$ becomes even more sensitive to length increases in small individuals, and less sensitive for large individuals (fig. 4B).

\section{Effects of Temperature on Stochastic Dynamics}

The detailed results of the analysis of the stochastic model are presented in appendix D. Overall, changes in the variability of temperature has very small effects compared to changes in the mean. The overall environmental variance in population growth is actually more affected by the mean temperature (a positive effect) than by variability. The stochastic population growth rate $r_{s}$ increases with mean temperature (at only a slightly lower rate as the growth rate of the mean environment). In appendix D, we also show that the results from the previous section regarding the decomposition of thermal sensitivity of $\lambda$ represent a good approximation also for the stochastic growth rate $\lambda_{s}$.

\section{Discussion}

In this study we have shown that warming is expected to increase the population growth rate of pike, but the underlying mechanisms are highly complex as the thermal sensitivity of the population growth rate varies across vital rates and body length (fig. $4 A$ ). Warming, measured in this study as an increase in the annual mean temperature, induces a shift in the stable length structure of the population toward an increased proportion of medium-sized pike and a reduced proportion of small and large pike. At the same time the somatic growth of individual pike follows the temperature-size rule, with increased growth rate at smaller lengths and a reduced asymptotic length. The environmental stochasticity in the population growth increases with warming but not enough to reduce the stochastic growth rate, which increases with temperature at almost the same rate as the growth rate in the mean environment. Changes in the variability of temperature had very small effects compared to changes in the mean temperature.

Temperature and body size are key determinants of individual vital rates in most organisms. Our results show that small and large pike show greatly different responses to temperature (fig. 1). Important physiological and biochemical processes are generally optimized within a certain thermal window, outside which functional constraints apply and fitness declines (Pörtner and Farrell 2008). The thermal sensitivity of an individual, which determines the size of this thermal window, can change throughout its life history, and fish are likely to be less sensitive at medium sizes than at small and large sizes (Pörtner and Knust 2007; Pörtner and Farrell 2008). For this pike population, we found that the somatic growth rate shows the largest temperature response at medium lengths (fig. 1C), perhaps reflecting changes in thermal sensitivity with size. This may occur because medium-sized individuals experience few negative effects of extreme temperatures, compared to large and small ones. Large individuals require more oxygen and may therefore be more sensitive to heat, while small individuals that are still in early development may be sensitive to both cold and warm temperatures (Pörtner and Farrell 2008).

Besides such direct physiological effects, temperature may also have indirect effects on the vital rates, through (i) changes in activity levels and behavior (Casselman 1996; Diana 1996); (ii) ecosystem changes, for instance, in the relative abundances of prey or competitor species (Winfield et al. 2008; Ohlberger 2013); or (iii) correlations with other vital rates that are affected by temperature, reflecting trade-offs or constraints in the life history. Altered activity levels can affect survival through different exposures to predation and competitors and increased lev- 

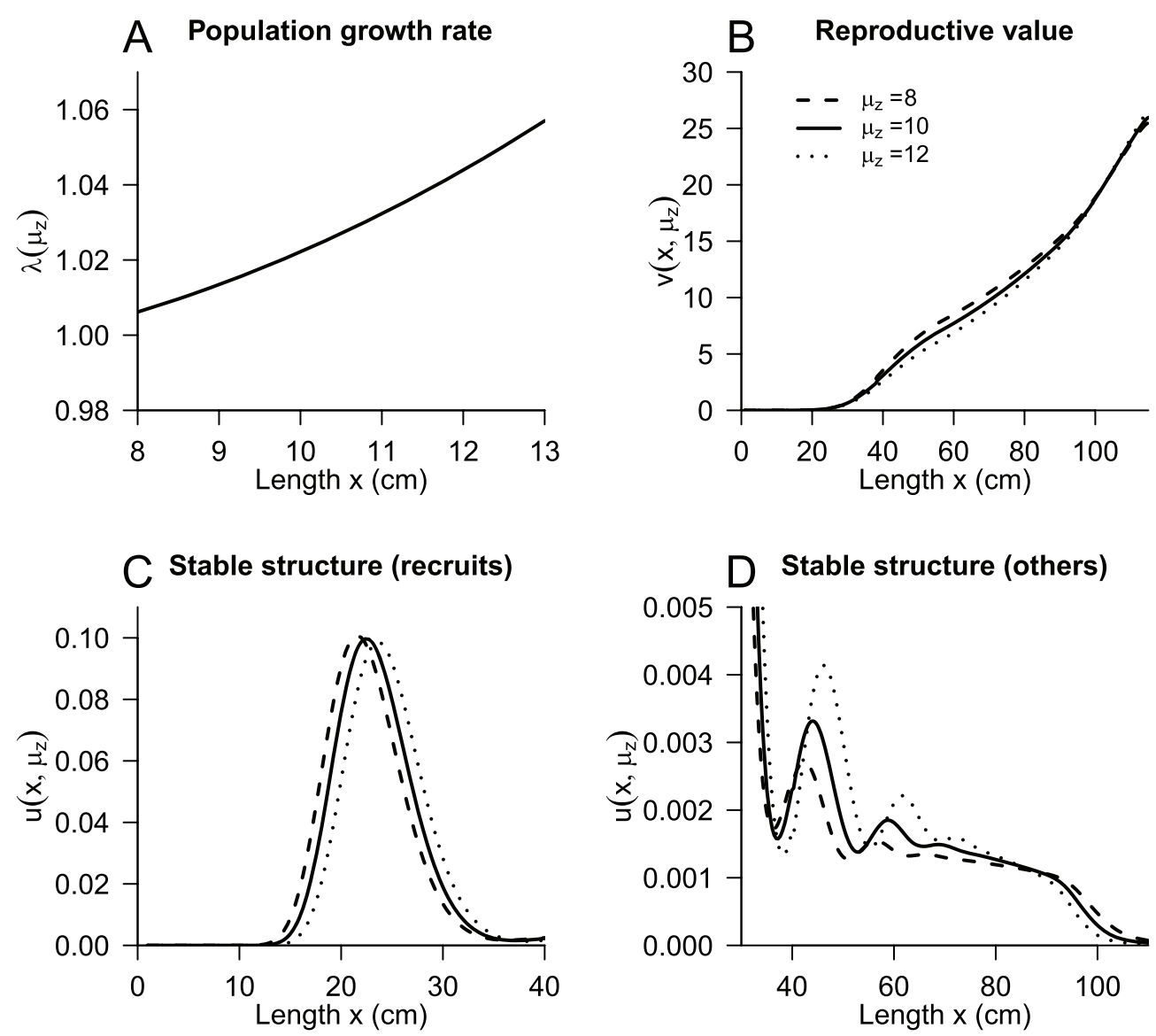

Figure 3: $A$, Population growth rate in the mean environment, as a function of temperature. Vertical lines indicate the mean temperatures used for calculation in $B-D$. B, Reproductive value as function of length at three temperatures. $C, D$, Stable length distribution at the three different temperatures (note different values on axes). Numerical calculations were performed using a mesh size of $n=300$ ( $\Delta x \approx 0.38$ $\mathrm{cm})$.

els of stress. Ecosystem effects through prey may not have as large consequences for pike as for a specialist predator, as pike are opportunistic and can switch between different prey species (Casselman 1996). The third possibility of correlated effects seem likely in this population, as the vital rates of pike are highly correlated in their response to warming (fig. 1). Such correlations may result from tradeoffs in energy investments throughout life history (Roff 2002). An optimal life history generally reflects optimal resource allocation into somatic growth and reproduction throughout life, in a way that maximizes the expected reproductive output over the lifetime (Charlesworth 1994; Kozlowski et al. 2004). If some vital rate changes as a result of warming, individuals can respond by changing the relative energy investments, so that the other vital rates also become affected. Thus, universal responses to climate change, such as the temperature-size rule in ectotherms, may result from adaptive responses (Kozlowski et al. 2004; Arendt 2010).
The TSR in ectotherms has been demonstrated across several taxa (Angilletta 2009), and average growth of pike in this population also seems to follow this pattern (fig. 2). While the TSR may reflect pure biochemical and physiological responses of the somatic growth process to temperature (Brown 2004), our results also suggest a possible adaptive explanation through trade-offs between somatic growth and fecundity, together with increased mortality (fig. 1). When survival is reduced due to warming, the expected reproductive life span of an individual decreases. Given that there is a trade-off between reproduction and somatic growth, individuals should then increase their reproductive investment compared to growth (Kozlowski et al. 2004). These results (fig. 1) indicate a response to warming where pike invest relatively more in growth than fecundity at small sizes and more in fecundity at larger sizes where mortality increases with temperature. In a theoretical model, Arendt (2010) showed that increased sizespecific fecundity in cold conditions may contribute to 

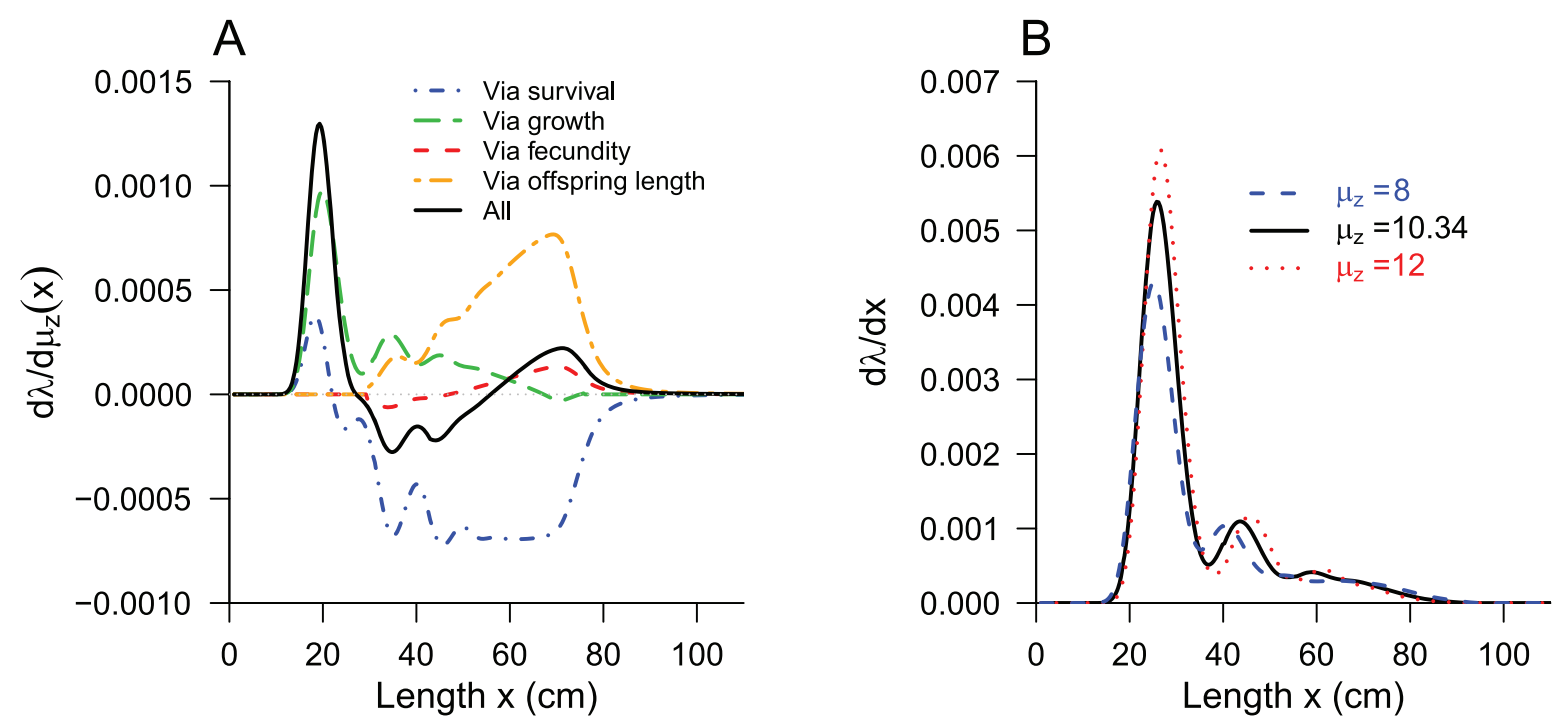

Figure 4: $A$, Length-specific contributions to the thermal sensitivity of the population growth rate, $\mathrm{d} \lambda / \mathrm{d} \mu_{z}=0.0101$ (calculated at $\mu_{z}=$ 10.34), from each of the four vital rate functions. The total contribution across length is also shown. The decomposition of $\mathrm{d} \lambda / \mathrm{d} \mu_{z}$ is given by equation (2), and $\mathrm{R}$ code, available online, is provided for calculation of the thermal sensitivity from the vital rates. $B$, Sensitivity of $\lambda$ to length $x$ (eq. [3]), for three different temperatures.

explain the temperature-size rule, as individuals could achieve the same fecundity level as in warm conditions at a lower body size. This is not the case in this pike population, as size-specific fecundity increases in warm rather than cold conditions (fig. 1A).

Our results also show a positive selection pressure for increased early growth (at all temperatures), but the selection is stronger in warm conditions (fig. $4 B$ ). Rapid growth is beneficial for small individuals, as it reduces the time exposed to low survival rates and allows the individual to reach the size of maturity earlier. A pattern of low survival at birth and increasing survival with size is common for many species, in particular for life histories with high fecundity and low parental investment (Roff 2002). The elevated selection pressure of the vital rates of small individuals in warm conditions may be explained by the increased population growth rate in warm conditions, favoring early life-history traits (Charlesworth 1994). Thus, while there is a large fitness benefit of rapid early growth at all temperatures, in terms of increased survival as well as reduced time to reproductive maturity, under warm conditions the accelerated population growth rate makes rapid early growth even more beneficial.

We made some important assumptions in this model: (i) that survival is not changing with length for large individuals (it still changes with temperature), (ii) that egg survival is independent of temperature, and (iii) that the population dynamics are density independent. We have shown that our results are qualitatively robust to the first two assumptions, except for the shape of the reproductive value function, which can show a dip at large lengths if survival declines for large individuals. Density dependence can be an important factor for some of the vital rates (Pierce et al. 2003), and this remains to be included in future developments of the model. Cannibalism, in particular, has been suggested as a potentially stabilizing mechanism for the size structure of pike (Persson et al. 2007). It affects the survival of smaller individuals negatively, possibly adding to the selection pressure for fast growth at small sizes (Edeline et al. 2007; Ohlberger et al. 2011a). In a recent model for perch that included cannibalism, a shift in age structure was observed, caused by a reduced adult survival and a subsequent increase in fecundity and juvenile survival (Ohlberger et al. 2011b). An interesting extension of our model would be to include density-dependent mortality from cannibalism depending on the length structure of the population and investigate under which conditions cannibalism may stabilize the length structure, as well as how cannibalistic interactions will be affected by climate warming.

In this study, changes in the interannual variability of temperature had much smaller consequences than changes in mean temperature, both for the mean population growth rate and for $\mathrm{n}$ environmental variance (app. D). A smaller effect of variability has also been reported in other demographic studies, although it can still be substantial (Jonzén et al. 2010; van de Pol et al. 2010). This result does not mean that stochasticity is not important 
for the population dynamics of pike, just that variability in temperature explains a small part of the overall variability in population growth. Our model also included residual environmental variation and covariation among vital rates, arising from other sources. This study did not consider changes in the intraannual variability, which may potentially have larger consequences than interannual variability. Evaluating consequences of temperature variability at different temporal scales is therefore another interesting topic for future studies.

This study demonstrates that demographic modeling can be useful in revealing complex underlying mechanisms for general responses to climate change, such as increased population growth and the temperature-size rule. The approach of integral projection modeling combines the power of statistical regression modeling with the analytical tractability of demographic models. However, demographic approaches to study climate change effects requires long-term individual-based data as well as observations of the relevant climate variables. The studies should be long enough that a wide range of environmental conditions, including rare ones, are included among the observations. Unfortunately, this type of data is not available for most species, in particular those of conservation concern. The existing long-term demographic time series, such as the time series for pike and other species in Windermere (Craig 1996), are therefore highly valuable (Clutton-Brock Sheldon 2010).

\section{Acknowledgments}

We are grateful to the many individuals who have participated in the Windermere data collection over the years and to the Freshwater Biological Association for their joint stewardship of these invaluable long-term data. Two reviewers provided helpful comments. This work was supported by Centre for Ecological and Evolutionary Synthesis, the Research Council of Norway (NORKLIMA), and the Natural Environment Research Council of the United Kingdom.

\section{Literature Cited}

Angilletta, M. J. 2009. Thermal adaptation. Oxford University Press, Oxford.

Arendt, J. D. 2010. Size-fecundity relationships, growth trajectories, and the temperature-size rule for ectotherms. Evolution 65:43-51.

Atkinson, D. 1994. Temperature and organism size-a biological law for ectotherms? Advances in Ecological Research 25:1-58.

Atkinson, D. 1996. Ectotherm life history responses to developmental temperatures. Pages 183-204 in I. A. Johnston and A. F. Bennett, eds. Animals and temperature: phenotypic and evolutionary adaptation. Cambridge University Press, Cambridge.

Bates, D., M. Maechler, and B. Bolker. 2011. lme4: linear mixedeffects models using S4 classes. R package version 0.999375-42. http://R-project.org/.

Bonner, J. T. 2006. Why size matters. Princeton University Press, Princeton, NJ.

Boylan, P., and C. E. Adams. 2006. The influence of broad scale climatic phenomena on long term trends in Atlantic salmon population size: An example from the River Foyle, Ireland. Journal of Fish Biology 68:276-283.

Brown, J. H. 2004. Toward a metabolic theory of ecology. Ecology 85:1771-1789.

Burnham, K. P., and D. R. Anderson. 2002. Model selection and multimodel inference: a practical information-theoretic approach. $2 d$ ed. Springer, New York, pp. 70-72.

Calder, W. A. 1984. Size, function and life history. Harvard University Press, Cambridge, MA.

Carlson, S. M., E. Edeline, A. Vøllestad, T. O. Haugen, I. J. Winfield, J. M. Fletcher, J. B. James, and N. C. Stenseth. 2007. Four decades of opposing natural and human-induced artificial selection acting on Windermere pike (Esox lucius). Ecology Letters 10:512-521.

Casselman, J. M. 1996. Age, growth and environmental requirements of pike. Pages 69-101 in J. F. Craig, ed. Pike: biology and exploitation. Chapman \& Hall, London.

Caswell, H. 2001. Matrix population models. 2nd ed. Sinauer, Sunderland, MA.

Caswell, H., R. M. Nisbet, A. M. De Roos, and S. Tuljapurkar. 1997. Structured-population models: many methods, a few basic concepts. Pages 3-17 in S. Tuljapurkar and H. Caswell, eds. Structuredpopulation models in marine, terrestrial, and freshwater systems. Chapman \& Hall, New York.

Charlesworth, B. 1994. Evolution in age-structured populations. 2nd ed. Cambridge University Press, Cambridge.

Clarke, A. 2003. Costs and consequences of evolutionary temperature adaptation. Trends in Ecology and Evolution 18:573-581.

Clutton-Brock, T., and B. C. Sheldon. 2010. Individuals and populations: the role of long-term, individual-based studies of animals in ecology and evolutionary biology. Trends in Ecology and Evolution 25:562-573.

Coulson, T., E. A. Catchpole, S. D. Albon, B. J. T. Morgan, J. M. Pemberton, T. Clutton-Brock, M. Crawley, and B. Grenfell. 2001. Age, sex, density, winter weather, and population crashes in Soay sheep. Science 292:1528-1531.

Craig, J. F. 1996. Pike: biology and exploitation. Chapman \& Hall, London.

Craig, J. F., and C. Kipling. 1983. Reproduction effort versus the environment: case histories of Windermere perch, Perca fluviatilis L., and pike, Esox lucius L. Journal of Fish Biology 22:713-727.

Crozier, L. G., A. P. Hendry, P. W. Lawson, T. P. Quinn, N. J. Manua, J. Battin, R. G. Shaw, and R. B. Huey. 2008. Potential responses to climate change in organisms with complex life histories: evolution and plasticity in Pacific salmon. Evolutionary Applications 1:252-270.

Daufresne, M., K. Lengfellner, and U. Sommer. 2009. Global warming benefits the small in aquatic ecosystems. Proceedings of the National Academy of Sciences of the USA 106:12788-12793.

Diana, J. S. 1996. Energetics. Pages 103-124 in J. F. Craig, ed. Pike: biology and exploitation. Chapman \& Hall, London.

Easterling, M. R., S. P. Ellner, and P. M. Dixon. 2000. Size-specific 
sensitivity: applying a new structured population model. Ecology 81:694-708.

Edeline, E., S. M. Carlson, L. C. Stige, I. J. Winfield, J. M. Fletcher, J. B. James, T. O. Haugen, A. Vøllestad, and N. C. Stenseth. 2007. Trait changes in a harvested population are driven by a dynamic tug-of-war between natural and harvest selection. Proceedings of the National Academy of Sciences of the USA 104:15799-15804.

Ellner, S. P., and M. Rees. 2006. Integral projection models for species with complex demography. American Naturalist 167:410-428.

Engen, S., R. Lande, B.-E. Sæther, and M. Festa-Bianchet. 2007. Using reproductive value to estimate key parameters in density-independent age-structured populations. Journal of Theoretical Biology 244:308-317.

Estes, J. A., J. Terborgh, J. S. Brashares, M. E. Power, J. Berger, W. J. Bond, S. Carpenter, et al. 2011. Trophic downgrading of planet earth. Science 333:301-306.

Friedland, K. D., and C. D. Todd. 2012. Changes in northwest Atlantic arctic and subarctic conditions and the growth response of Atlantic salmon. Polar Biology 35:593-609.

Frost, W. E., and C. Kipling. 1959. The determination of the age and growth of pike (Esox lucius 1.) from scales and opercular bones. ICES Journal of Marine Science 24:314-341.

. 1967. A study of reproduction, early life, weight-length relationship and growth of pike, Esox lucius 1., in Windermere. nal of Animal Ecology 36:651-693.

Gardner, J. L., A. Peters, M. R. Kearney, L. Joseph, and R. Heinsohn. 2011. Declining body size: a third universal response to warming? Trends in Ecology and Evolution 26:285-291.

Haccou, P., P. Jagers, and V. A. Vatutin. 2005. Branching processes: variation, growth and extinction of populations. Cambridge University Press, Cambridge.

Haugen, T. O., I. J. Winfield, A. Vøllestad, J. M. Fletcher, J. B. James, and N. C. Stenseth. 2007. Density dependence and density independence in the demography and dispersal of pike over four decades. Ecological Monographs 77:483-502.

Hunter, C. M., H. Caswell, M. C. Runge, E. V. Regehr, S. C. Amstrup, and I. Stirling. 2010. Climate change threatens polar bear populations: a stochastic demographic analysis. Ecology 91:2883-3897.

IPCC (Intergovernmental Panel on Climate Change). 2007. Climate change 2007: synthesis report. Contributions of working groups I, II, and III to the Fourth Assessment Report of the IPCC. IPCC, Geneva.

Jenkins, G. J., J. M. Murphy, D. M. H. Sexton, J. A. Lowe, P. Jones, and C. G. Kilsby. 2009. UK climate projections: briefing report. Technical report. Met Office Hadley Center, Exeter, United Kingdom.

Jenouvrier, S., H. Caswell, C. Barbraud, M. Holland, J. Stroeve, and H. Weimerskirch. 2009. Demographic models and IPCC climate projections predict the decline of an emperor penguin population. Proceedings of the National Academy of Sciences of the USA 106: 1844-1847.

Jonsson, B., and N. Jonsson. 2009. A review of the likely effects of climate change on anadromous Atlantic salmon Salmo salar and brown trout Salmo trutta, with particular reference to water temperature and flow. Journal of Fish Biology 75:2381-2447.

Jonzén, N., T. Pople, J. Knape, and M. Sköld. 2010. Stochastic demography and population dynamics in the red kangaroo Macropus rufus. Journal of Animal Ecology 79:109-116.

Kipling, C., and W. E. Frost. 1970. A study of the mortality, population numbers, year class strengths, production and food con- sumption of pike, Esox lucius L., in Windermere from 1944 to 1962. Journal of Animal Ecology 39:115-157.

Kipling, C., and E. D. Cren. 1984. Mark-recapture experiments on fish in Windermere, 1943-1982. Journal of Fish Biology 24:395414.

Kozlowski, J., M. Czarnoleski, and M. Danko. 2004. Can optimal resource allocation models explain why ectotherms grow larger in cold? Integrative and Comparative Biology 44:480-493.

Lagler, K. F. 1978. Capture, sampling and examination of fishes. Pages 7-47 in T. B. Bagenal, ed. Methods for assessment of fish production in fresh waters. 3rd ed. Blackwell Scientific, Oxford.

Lande, R., and S. H. Orzack. 1988. Extinction dynamics of agestructured populations in a fluctuating environment. Proceedings of the National Academy of Sciences of the USA 85:7418-7421.

Langangen, Ø., E. Edeline, J. Ohlberger, I. J. Winfield, J. M. Fletcher, J. B. James, N. C. Stenseth, and A. Vøllestad. 2011. Six decades of pike and perch population dynamics in Windermere. Fisheries Research 109:131-139.

Le Cren, D. 2001. The Windermere perch and pike project: an historical review. Freshwater Forum 15:3-34.

Metz, J. A. J., and O. Diekmann. 1986. The dynamics of physiologically structured populations. Springer, Berlin.

Ohlberger, J. 2013. Climate warming and ectotherm body size: from individual physiology to community ecology. Functional Ecology 27:991-1001. doi:10.1111/1365-2435.12098.

Ohlberger, J., E. Edeline, A. Vøllestad, N. C. Stenseth, and D. Claessen. 2011a. Temperature-driven regime shifts in the dynamics of size-structured populations. American Naturalist 177:211-223.

Ohlberger, J., Ø. Langangen, E. Edeline, D. Claessen, I. J. Winfield, N. C. Stenseth, and L. A. Vøllestad. 2011b. Stage-specific biomass overcompensation by juveniles in response to increased adult mortality in a wild fish population. Ecology 92:2175-2182.

Parmesan, C. 2006. Ecological and evolutionary responses to recent climate change. Annual Review of Ecology, Evolution, and Systematics 37:637-669.

Peck, L. S., M. S. Clark, S. A. Morley, A. Massey, and H. Rossetti. 2009. Animal temperature limits and ecological relevance: effects of size, activity and rates of change. Functional Ecology 23:248256.

Persson, L., P.-A. Amundsen, A. M. De Roos, A. Klemetsen, R. Knudsen, and P. Primicerio. 2007. Culling prey promotes predator recovery: alternative states in a whole-lake experiment. Science 316 : 1743-1746.

Pierce, R. B., C. M. Tomcko, and T. L. Margenau. 2003. Density dependence in growth and size structure of northern pike populations. North American Journal of Fisheries Management 23: 331-339.

Pinheiro, J., D. Bates, S. DebRoy, D. Sarkar, and the R Development Core Team. 2011. nlme: linear and nonlinear mixed effects models. R package version 3.1-102. http://R-project.org/.

Pörtner, H. O., and A. Farrell. 2008. Physiology and climate change. Science 322:690-692.

Pörtner, H. O., and R. Knust. 2007. Climate change affects marine fishes through oxygen limitation of thermal tolerance. Science 315 : 95-97.

R Development Core Team. 2011. R: a language and environment for statistical computing. R Foundation for Statistical Computing, Vienna.

Rees, M., and S. P. Ellner. 2009. Integral projection models for pop- 
ulations in temporally varying environments. Ecological Monographs 79:575-594.

Roff, D. A. 2002. Life history evolution. Sinauer, Sunderland, MA, pp. 198-210.

Savage, V. M., J. F. Gillooly, J. F. Brown, G. B. West, and E. L. Charnov. 2004. Effects of body size and temperature on population growth. American Naturalist 163:429-441.

Stearns, S. C. 2000. Life history evolution: successes, limitations, and prospects. Naturwissenschaften 87:476-486.

Stenseth, N. C., A. Mysterud, G. Ottersen, J. W. Hurrell, K.-S. Chan, and M. Lima. 2002. Ecological effects of climate fluctuations. $\underline{\text { Sci- }}$ ence 297:1292-1296.

Tuljapurkar, S. 1990. Population dynamics in variable environments. Springer, Berlin.

van de Pol, M., Y. Vindenes, B.-E. Sæther, S. Engen, B. J. Ens, K. Oosterbeek, and J. Tinbergen. 2010. Effects of climate change and variability on population dynamics in a long-lived shorebird. Ecology 91:1192-1204.

- 2011. Poor environmental tracking can make extinction risk insensitive to the colour of environmental noise. Proceedings of the Roval Society B: Biological Sciences 278:3713-3722.

Vindenes, Y., S. Engen, and B.-E. Sæther. 2011. Integral projection models for finite populations in a stochastic environment. Ecology 92:1146-1156.

Visser, M. E., and C. Both. 2005. Shifts in phenology due to global climate change: the need for a yardstick. Proceedings of the Roval Society B: Biological Sciences 272:2561-2569.

Walther, G. R., E. Post, P. Convey, A. Menzel, C. Parmesan, T. J. C. Beebee, J. M. Fromentin, O. Hoegh-Guldberg, and F. Bairlein. 2002. Ecological responses to recent climate change. Nature 416: 389-395.

Williams, S. E., L. P. Shoo, J. L. Isaac, A. A. Hoffmann, and G. Langham. 2008. Towards an integrated framework for assessing the vulnerability of species to climate change. PLoS Biology 6: 2621-2626.
Winfield, I. J., and J. M. Fletcher. 2013. Windermere lake temperature data 1944-2002. NERC Environmental Information Data Centre. Accessed September 13, 2013. http://dx.doi.org/10.5285/9520664c -eb4d-4700-b064-5d215d23e595. doi:10.5285/9520664c-eb4d -4700-b064-5d215d23e595.

Winfield, I. J., J. M. Fletcher, and J. B. James. 2013a. Pike fecundity data 1963-2002. NERC Environmental Information Data Centre. Accessed September 13, 2013. http://dx.doi.org/10.5285 /b8886915-14cb-44df-86fa-7ab718acf49a. doi:10.5285/b8886915 -14cb-44df-86fa-7ab718acf49a.

- 2013b. Pike growth data 1944-1995. NERC Environmental Information Data Centre. Accessed September 13, 2013. http:// dx.doi.org/10.5285/637d60d6-1571-49af-93f7-24c1279d884d. doi: 10.5285/637d60d6-1571-49af-93f7-24c1279d884d.

- 2013c. Pike survival data 1953-1990. NERC Environmental Information Data Centre. Accessed September 13, 2013. http:// dx.doi.org/10.5285/813e07dd-2135-49bc-93c6-83999e442b36. doi: 10.5285/813e07dd-2135-49bc-93c6-83999e442b36.

Winfield, I. J., J. B. James, and J. M. Fletcher. 2008. Northern pike (Esox lucius) in a warming lake: changes in population size and individual condition in relation to prey abundance. Hydrobiology 601:29-40.

Wright, R. M. 1990: The population biology of pike, Esox lucius L., in two gravel pit lakes, with special reference to early life history. Journal of Fish Biology 36.215-229.

Zuur, A. F., E. N. Ieno, N. J. Walker, A. A. Saveliev, and G. M. Smith. 2009. Mixed effects models and extensions in ecology with R. Statistics for biology and health. Springer Science and Business, New York.

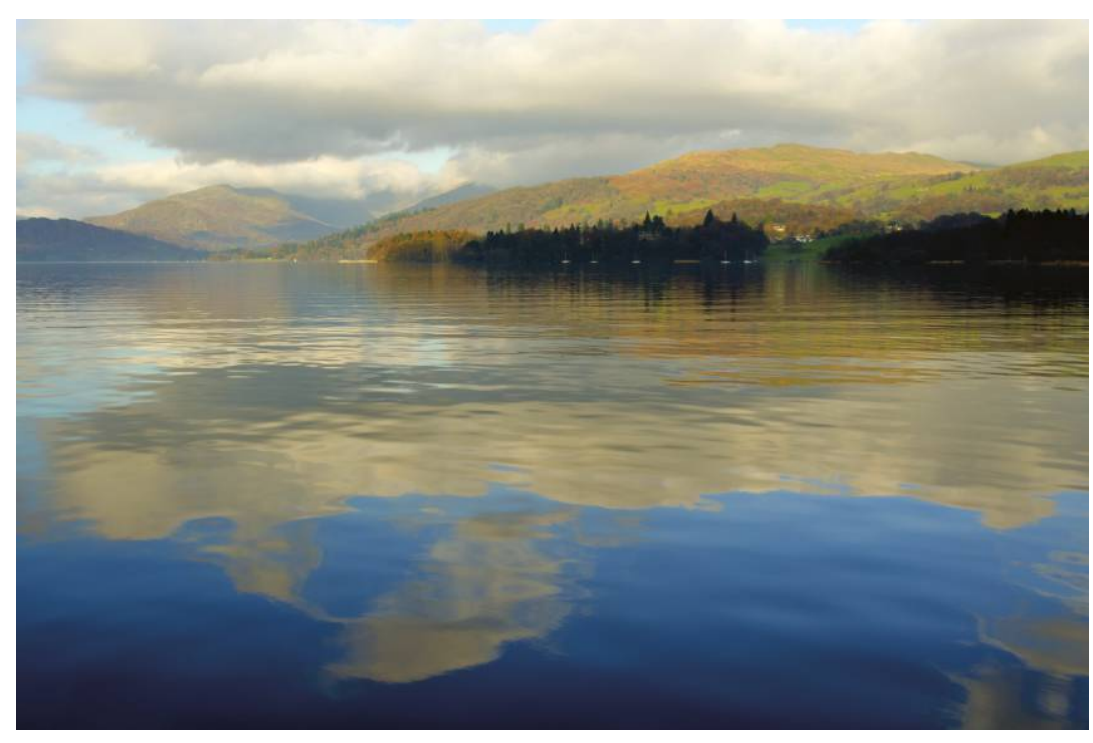

Windermere, home of the northern pike (Esox lucius) population under study. Photo credit: Ian J. Winfield. 